\title{
CoviD-19 Managing high-acuity outpatient services during the COVID-19 pandemic: lessons from the acute diabetes foot service
}

\author{
Authors: Gurdev S Deogon, ${ }^{\mathrm{A}}$ Tim Robbins, ${ }^{\mathrm{B}}$ Manpal S Randeva, ${ }^{\mathrm{C}}$ Ioannis Kyrou, ${ }^{\mathrm{D}}$ Sailesh Sankar, ${ }^{\mathrm{E}}$ Harpal S Randeva ${ }^{\mathrm{F}}$ \\ and Narasimha Murthy ${ }^{G}$
}

\begin{abstract}
COVID-19 has created unprecedented challenges for healthcare services internationally. Many NHS organisations have cancelled outpatient clinics to release frontline clinical staff and minimise risk of patients contracting COVID-19. While many outpatient services manage chronic diseases, a number of services manage high-acuity patients. Delivery of these acute outpatient services during the pandemic has posed particular challenges and required significant service model reconfiguration. The acute diabetes foot clinic is an important example of such a service. We explore the important lessons learnt during the COVID-19 pandemic for managing high-acuity outpatient services through the context of the diabetic foot clinic. Learning can be divided into the following categories: remote and digital working, physical changes in service delivery, workforce challenges and post-pandemic preparedness. This learning is applicable to a wide range of high-acuity services during and following the pandemic. It is particularly relevant as we expand outpatient care provision to avoid hospital admissions.
\end{abstract}

KEYWORDS: Outpatient care, diabetic foot disease, diabetes, COVID-19

DOI: $10.7861 /$ fhj.2020-0054

Authors: ${ }^{A}$ chief podiatric specialist in diabetes, Warwickshire Institute for the Study of Diabetes, Endocrinology and Metabolism (WISDEM), Coventry, UK; ${ }^{\text {B }}$ medical registrar, WISDEM, Coventry, UK and University of Warwick, Coventry, UK; Cmedical student, WISDEM, Coventry, UK and University College London, London, UK; ${ }^{D}$ Clinical lecturer and diabetes and endocrinology fellow, WISDEM, Coventry, UK, Aston University, Birmingham, UK and University of Warwick, Coventry, UK; Eassociate director of medical education and consultant in diabetes and endocrinology, WISDEM, Coventry, UK and University of Warwick, Coventry, UK; Fhead and clinical lead for diabetes, endocrinology and metabolism, WISDEM, Coventry, UK and University of Warwick, Coventry, UK; ${ }^{G}$ Consultant and lead for diabetic foot service, WISDEM, Coventry, UK

\section{Introduction}

Healthcare services internationally have cancelled the majority of elective activity to focus on emergency care during the novel coronavirus (COVID-19) pandemic. This has had significant impact on the majority of outpatient clinic services. While many outpatient services manage chronic disease conditions, a number of services manage high-acuity patients on an outpatient basis. An important example of this is the outpatient management of people with acute and severe presentation of diabetes foot disease. Overall, it is well recognised that diabetes foot syndrome is associated with high levels of morbidity and premature mortality. ${ }^{1}$ As such, 5-year mortality is $>50 \%$ in diabetes patients with foot ulcers, which is similar to or worse than many common cancers. ${ }^{2}$ Failure to manage this cohort of patients appropriately during the COVID-19 pandemic has the potential for profound morbidity and mortality. Conversely, these patients are also at very high risk from COVID-19 infection. ${ }^{3}$ It is essential that contact with these patients is minimised to support the shielding of vulnerable patients. Therefore, set within the context of a healthcare system in an unprecedented crisis, conflicting demands currently exist for intensively managing diabetic foot disease, while also minimising risk of exposure to COVID-19.

This article explores both the challenges and potential solutions of managing high-acuity outpatient services through the perspective of the Warwickshire Institute for the Study of Diabetes, Endocrinology and Metabolism (WISDEM) acute diabetes foot service. WISDEM is a specialist centre providing treatment and support for the more complex cases of diabetes, endocrine and metabolic conditions in the UK West Midlands region. The interdisciplinary foot-care team serves the active and advanced stage foot needs of the city-wide integrated foot-care pathway and receives wider tertiary referrals. In line with ambitions provided in national guidance, ${ }^{4}$ key strategic aims are intended to reduce rates of hospital admission, length of stay and lower limb amputations (LLA).

\section{The COVID-19 pandemic and diabetic foot disease}

Prior to the World Health Organization (WHO) declaring the current COVID-19 outbreak a pandemic on 11 March 2020, ${ }^{5}$ the General Medical Council (GMC) focused awareness on the gravitas of COVID-19 on 3 March 2020. 6 On 16 March 2020, NHS England provided specialty guidance for acute diabetes care, advocating 


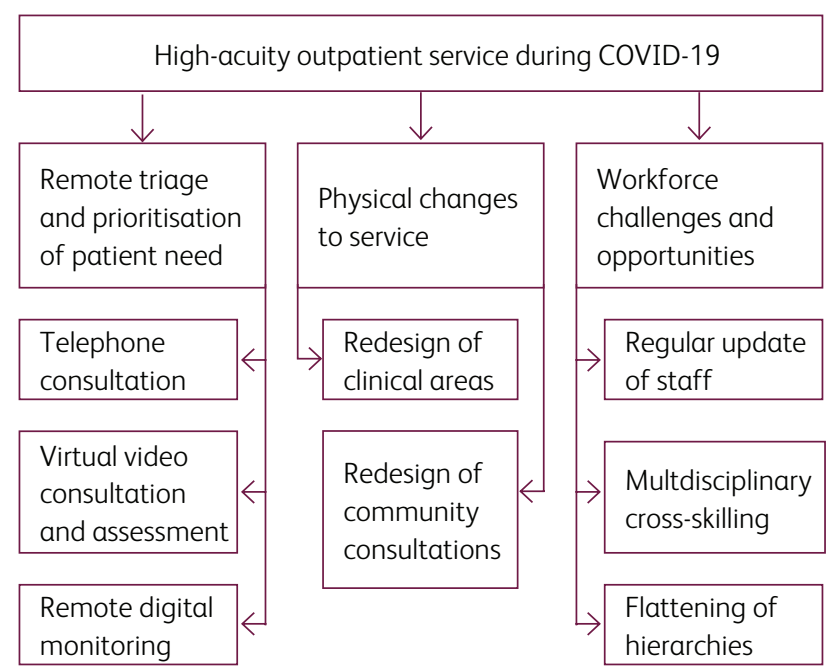

Fig 1. Summary of changes to acute diabetic foot service during COVID-19.

that multidisciplinary diabetes foot services should continue at full capacity, owing to acuity and morbidity risks. ${ }^{7}$ As the UK 'lockdown' was announced on 23 March 2020, further guidance on LLA prevention during the COVID-19 situation followed from Foot in Diabetes UK (FDUK), which focused on clinical assessment and key decision making for life- and limb-saving treatment. ${ }^{8}$ Delivering high-quality care for people with acute diabetes foot disease in the pandemic setting required significant and rapid change to service design and delivery. Those changes can broadly be split into four main areas: remote/digital working, physical changes in service delivery models, workforce challenges/solutions and post-pandemic preparedness. These are presented graphically in Fig 1; however, it is essential to recognise the significant interdependence between these different parts, with none being sufficient to deliver high-quality care in isolation.

\section{Remote and digital working}

Early stages of the COVID-19 pandemic progressed rapidly, requiring clinical and operational teams to be responsive; this required constant review and optimisation of service delivery. In line with the national effort to reduce disease transmission, a key facilitator at the WISDEM Foot Service was early adoption of virtual remote consultation to assist and prioritise foot clinic attendance based on clinical need.

Communication through this new virtual clinic model was enabled through digital technology. Diabetes foot care requires visual assessment of disease, making it different from many other chronic condition consultations. Image-based digital tools are therefore required. Importantly, while evidence shows that there can be interpersonal subjectivity of interpreting images, the information harnessed from these visual sources serves as a feasible adjunct to decision making and planning in the foot-care setting. ${ }^{9}$

Utilisation of a digital application 'Secure Clinical Image Transfer' (SCIT) and bespoke 'Medopad' remote consultation tool for diabetes foot disease has enabled image capture, storage, remote consultation and electronic patient record linkage. Together, these tools allow patients and clinicians to keep and securely share photographic records of foot disease using a personal smartphone device, while supporting the tracking, monitoring and remote management of foot conditions. Pilot implementation in the WISDEM acute diabetes foot clinic has received national recognition; it was recently selected as a Health Service Journal Value Award finalist.

WISDEM patients requiring advanced lower limb unloading devices (ranging from removable soft casts to patellar tendonbearing orthoses) are able to provide targeted video content, filmed on their own devices and used for gait analysis and orthosis fitting. This assists in selection of patients who would benefit from supply and fitting of alternative provision or minor adjustment through 'doorstep' visits.

\section{Physical changes in service delivery models}

Effective digital transformation cannot be achieved in isolation, and wider supportive and adaptive changes are required. This approach is no different in response to the COVID-19 pandemic. Clinic spaces are required to make physical adaptations to comply with social distancing measures, with direct impacts on patient flow pathways, requiring staggering of appointments and reconfiguration of waiting room seating.

Diabetes foot disease community visits have been redesigned as 'doorstep' visits, minimising contact and exposure to both patients and healthcare staff. An effective 'doorstep' visit (where the clinician remains outside the home) requires careful preparation and reflects a new model of clinical consultation. Importantly, doorstep visits provide significant reassurance to patients who are often reluctant to seek or engage with healthcare staff for fear of contracting COVID-19.

Minimising staff exposure has similarly been essential, and reassurance for this group is also needed. Staff briefings regarding the use of personal protective equipment (PPE) including continual updates based on Public Health England guidance, ${ }^{10}$ with advanced monitoring of availability, its timely procurement and correct level of issue for respective foot team personnel, including those in the community setting, have been essential to continued success of the service.

Overall, there have been significant changes in the pathways/ consultations for patients attending this high-acuity outpatient service. As expected in such services, patient-tailored approaches/ pathways are followed to manage each patient's complex disease and comorbidities. In this context, under the current pandemic circumstances, a typical new patient is initially telephone triaged, following receipt of the corresponding digital referral. Subsequently, the majority of these new-patient triages result in a face-to-face consultation being planned, with appropriate social distancing and timings between consultations. During the initial face-to-face consultation, digital image capture will record a benchmark of the existing foot disease and the patient will be introduced to the virtual and remote monitoring tools used by the clinic. Moreover, regular follow-up now consists of virtual consultation models for stable or improving disease, instead of the previously scheduled separate, face-to-face consultations with both the medical and orthotist team. As such, orthotist fitting and gait analysis are now delivered for these patients through a combination of self-filmed targeted video content and doorstep fitting and adjustment. In this way, patients also have increased empowerment to contact the clinic outside the usual booked clinic sessions and there is a more collaborative approach to managing 
the patient safely between themselves, their family/carers and the multidisciplinary teams.

The impact of these digital and physical changes will need to be tracked through audit and quality assurance processes over the next 12 months. From reviewing local clinic lists, we have identified that comparing the March-May period of 2019 to the same months of 2020, 16 patients, on average, were seen face to face per multidisciplinary clinic in 2019, compared with eight virtual and eight face-to-face consultations in 2020. A comprehensive local audit of the changes in our service is already planned for the following 12 months. Of note, there is also a National Diabetes Foot Care Audit (NDFA) as part of the established and wellrespected National Diabetes Audit Programme. ${ }^{11}$ Such local and national audits are exceptionally useful tools for benchmarking services in a high-acuity outpatient service and are expected to allow better understanding of the impact of COVID-19 on diabetes foot-care services and their patients.

\section{Workforce challenges and solutions}

Despite careful planning of PPE provision, COVID-19 presents wideranging workforce challenges due to staff sickness, self-isolation, redeployment, additional duties and staff mental wellbeing issues. The diabetes foot clinic represents a unique workforce challenge due to the multidisciplinary skills needed. In particular, it is essential that patients can access all services on one visit rather than having to make multiple visits due to staff unavailability. At the WISDEM centre, longstanding approaches to cross-skilling among the core multidisciplinary team have created resilience within the service. Problems can therefore be managed with an, at times, reduced workforce. Similarly, cross-skilling outside the core team, with initiatives such as rescue pack antibiotics and liaison with the pharmacists, helps to support continuity and provision of treatment. This cross-skilling approach has resulted in a flattening of traditional hierarchies, a phenomenon that we expect to see continuing long after the COVID-19 pandemic has passed.

\section{Preparing NHS acute diabetic foot care for the post-pandemic phase}

The COVID-19 pandemic continues to present challenges, and it is essential that service providers remain adaptive. The decline in hospital visits for patients with non-limb-threatening (deemed important but not urgent) diabetes foot disease inevitably has the potential to spill over as a tsunami of diabetic foot disease resurgence in a post-COVID-19 scenario. Optimal preparedness for this is essential, and clearly reliant on ensuring that service capacity in the future remains a key focus within operational planning. Equally, careful clinical prioritisation will be necessary to mitigate risk from surges of demand. At WISDEM, we are embarking on pre-emptive contacting of all diabetic foot patients to establish how they are managing in self-isolation, regardless of planned clinic visit date. This process has a strong focus on both physical and psychological health and will enable us to prioritise patients at greatest need of early support post-COVID-19 and to signpost to additional support needed during lockdown.

\section{Conclusions}

As the pandemic's consequential grip on society and normal life is rapidly being realised, the resulting changes may well redefine management of high-acuity outpatient services as we currently know them. In particular, there appears to be a rapid erosion of any historical inertia to the integration of digital technologies and novel approaches to healthcare delivery. As teams progressively address the challenges on both a national and a local level, measures of responsiveness and openness to trial novel approaches will determine the measure and scale of morbidity and mortality end points. The flattening of traditional hierarchies and cross-discipline working are important changes that may have lasting sustained benefits to multidisciplinary teams. While our primary goal remains to minimise the impact on patients and their quality of life, embracing this opportunity as a positive learning experience may significantly reshape the way we manage this vulnerable caseload in the future.

\section{References}

1 Chammas NK, Hill RLR, Edmonds ME. Increased mortality in diabetic foot ulcer patients: the significance of ulcer type. J Diabetes Res 2016;2016:2879809.

2 Armstrong DG, Wrobel J, Robbins JM. Guest editorial: are diabetes-related wounds and amputations worse than cancer? Int Wound J 2007:4:286-7.

3 Fang L, Karakiulakis G, Roth M. Are patients with hypertension and diabetes mellitus at increased risk for COVID-19 infection? Lancet Respir Med 2020;8:e21.

4 National Institute for Health and Care Excellence. Diabetic foot problems: prevention and management [NG19]. London: NICE, 2015. www.nice.org.uk/guidance/ng19 [Accessed 22 July 2020].

5 World Health Organization. WHO Director-General's opening remarks at the media briefing on COVID-19 - 11 March 2020. Geneva: WHO, 2020. www.who.int/dg/speeches/detail/who-director-general-s-opening-remarks-at-the-media-briefing-on-covid-1911-march-2020 [Accessed 22 July 2020].

6 General Medical Council. How we will continue to regulate in light of novel coronavirus. London: GMC, 2020. www.gmc-uk.org/news/ news-archive/how-we-will-continue-to-regulate-in-light-of-novelcoronavirus [Accessed 22 July 2020].

7 NHS England. Clinical guide for the management of people with diabetes during the coronavirus pandemic. London: NHS England, 2020. www.england.nhs.uk/coronavirus/wp-content/uploads/ sites/52/2020/03/speciality-guide-diabetes-19-march-v2-updated. pdf [Accessed 22 July 2020].

8 Foot in Diabetes UK. COVID-19 SITUATION v1.3 Lower Limb Amputation Prevention Guidance. Diabetic Foot J 2020;23:2. www.diabetesonthenet.com/journals/issue/618/article-details/ covid-19-situation-v13-lower-limb-amputation-prevention-guidance1 [Accessed 28 July 2020].

9 Hazenberg CEVB, aan de Stegge WB, Van Baal SG, Moll FL, Bus SA Telehealth and telemedicine applications for the diabetic foot: A systematic review. Diabetes Metabolism Res Rev 2020;36:e3247

10 Sayburn A. Covid-19: PHE upgrades PPE advice for all patient contacts with risk of infection. BMJ 2020;369:m1391.

11 Jeffcoate W, Gooday C, Harrington A, Lewis J, Cawley S, Young B. The National Diabetes Foot Care Audit of England and Wales: achievements and challenges. Diabetic Foot ] 2020;23:70-3.

Address for correspondence: Mr Gurdev Singh Deogon, University Hospital, University Hospitals Coventry and Warwickshire NHS Trust, Clifford Bridge Road, Coventry CV2 2DX, UK.

Email: gurdev.deogon@uhcw.nhs.uk 